\title{
The Australian Desert Fireball Network: A new era for planetary science
}

\author{
P. A. Bland ${ }^{1,2,3,5}$, G. K. Benedix ${ }^{2}$, A. W. R. Bevan ${ }^{\star 5}$, K. T. Howard ${ }^{2}$, P. Spurný ${ }^{4}$, \\ L. Shrbený ${ }^{1,4}$, M. C. Towner ${ }^{1}$, I. A. Franchi ${ }^{6}$ G. Deacon ${ }^{5}$, J. Borovička ${ }^{4}$, D. \\ Vaughan $^{7}$, R. M. Hough ${ }^{8}$.
}

${ }^{1}$ IARC, Department of Earth Science \& Engineering, Imperial College London, SW7 2AZ, UK.

${ }^{2}$ IARC, Dept. Mineralogy, Natural History Museum, London SW7 5BD, UK.

${ }^{3}$ Department of Applied Geology, Curtin University of Technology, GPO Box U1987, Perth WA 6845, Australia.

${ }^{4}$ Astronomical Institute of the Academy of Sciences, Fričova 298, CZ-251 65 Ondřejov Observatory, Czech Republic.

${ }^{5}$ Department of Earth and Planetary Sciences, Western Australian Museum, Locked Bag 49 Welshpool DC, WA 6986, Australia., Perth, WA 6845, Australia.

${ }^{6}$ PSSRI, Open University, Walton Hall, Milton Keynes MK7 6AA, UK. ${ }^{7}$ PO BOX 187, Nedlands, Perth, WA 6909, Australia.

8 CSIRO Earth Science and Resource Engineering, 26 Dick Perry Avenue, Kensington, Western Australia 6151.

Corresponding author: alex.bevan@museum.wa.gov.au 


\section{INTRODUCTION}

Meteorites are our only direct source of information on the nature of planetary materials in the early Solar System. Most meteorites are believed to be fragments broken from asteroids in solar orbits between Mars and Jupiter, although there are few specific asteroids identified as sources. Other meteorites are fragments from the Moon and Mars. Many asteroidal meteorites have remained virtually unaltered for $4.56 \mathrm{Ga}$ and retain evidence of the earliest formative processes of the Solar System, ranging from presolar stellar evolution of the nearby galactic region, to protoplanetary disk formation and the accretion and differentiation of planetesimals and protoplanets.

Of the more than 50000 meteorites now in collections around the world, the vast majority are chance finds (Bevan 2006). Over the last 300 years or so, world-wide only about 1100 meteorites have actually been observed to fall and quickly recovered (Grady 2000). Of these, the atmospheric phenomena associated with the fall of only 12 recovered meteorites have been photographed allowing the orbits of the objects that gave rise to the meteorites to be determined. To date, only 5 falls have been recorded by camera networks, the remainder were recorded by chance photography. Fragments of another meteorite fall were recovered by tracking a small asteroid that eventually collided with the Earth (Jenniskens et al. 2009). 
While there is no shortage of meteorites to study, the unique information they record has to be interpreted in the absence of the spacial context of where in the Solar System the material originated. What is lacking is a significant number of photographed falls with precisely calculated orbits. An expanded collection of meteorites with known orbits would allow the association of some samples to specific regions or bodies in the Solar System, providing the spatial context for the interpretation of meteorite composition.

The newly established Desert Fireball Network (DFN) in the Western Australian Nullarbor is a world-leading facility designed to provide fundamentally important information to planetary scientists about the nature and origin of meteorites. Through an international collaboration between Imperial College London, the Ondrejov Observatory in the Czech Republic and the Western Australian Museum in Perth, construction of a trial network comprising four Desert Fireball Observatories (DFOs) was completed in 2007 and achieved almost immediate success. A meteorite fall photographed in July 2007 was later recovered within 100 metres of the landing site predicted by the network (Bland et al. 2009).

\section{CAMERA NETWORKS}

Fireball camera networks providing precise triangulation of fireball records from multiple stations to constrain the fall positions and pre-atmospheric orbits of meteorites, have been in operation in various parts of the world for over 40 years (e.g. see Bowden 2006 and references therein). The two principal scientific objectives of these programmes were to provide an accurate 
estimate of the flux of extraterrestrial material to Earth, and to recover a significant number of meteorites with accurately determined orbits.

However, although these networks succeeded in constraining the meteorite flux over a range of masses (Halliday et al. 1989), and hundreds of fireballs potentially associated with the fall of meteorites were recorded, only four meteorites were recovered. The reason for this poor rate of recovery is that the camera networks were established in areas of the world (central Europe, USA and Canada) with terrain that is not generally conducive to the recognition of small meteorites on the ground.

A number of arid areas of the world have proved to be 'storehouses' of past meteorite falls, notably the wider Sahara in northern Africa, the Nullarbor Region in Australia, the deserts of Oman, and the cold desert of Antarctica (Bevan 2006; Zolensky, 1998). Prolonged aridity in these regions has allowed the preservation of meteorites over millenia and their accumulation on generally stable surfaces. These desert conditions and the general lack of vegetation allows the easy recognition and recovery of meteorites, including those that may be observed to fall.

In the mid 1990s, with seed funding from National Geographic, the Western Australian Museum, the Royal Society and the Open University, a feasibility study was undertaken to select a suitable desert area to establish a new camera network. For a variety of reasons including political stability, accessibility and relatively low fieldwork costs, the Nullarbor Region and other 
areas of the western desert of Australia proved ideal. Planning for a trial Desert Fireball Network based in the Nullarbor Region of Western Australia began in 1997. However, a suitably designed fireball observatory that could operate in harsh conditions and with minimal human intervention had first to be developed.

\section{CAMERA DEVELOPMENT, OPERATION AND DEPLOYMENT}

To satisfy the stringent requirements for a camera to operate auronomously in desert conditions, the Autonomous Fireball Observatory (AFO), a complex instrument for fireball observations developed for the European Fireball Network (Spurny et al. 2006) was modified for the purpose. The prototype Desert Fireball Observatory (DFO) (Figure 1) was completed in 2003 and installed for testing at a site to the east of Kalgoorlie. The site allowed access for maintenance, but with environmental conditions similar to that which would be experienced when the DFO was established on the Nullarbor.

The DFO weighs approximately $100 \mathrm{~kg}$, is weather resistant and hermetically sealed. The camera housing is coated with special paint that reflects ca. $85 \%$ of incident solar radiation. The lens and interior are kept at a constant temperature. A solar shield provides shade during the day time, and is retracted 30 mins before exposure time. The camera automatically takes exposures according to a prescribed schedule that is modified by prevailing weather conditions that are detected by the camera. The DFO incorporates a basic weather station and a sensitive CCD camera that monitors cloud cover. Climatic conditions are automatically evaluated before and during exposure. 
The information is processed digitally and continuously assessed. Exposure commences only under clement, clear conditions. From a GPS chip, the camera receives absolute date/time (Universal Time) signals, and the beginning and end of an exposure are recorded. An exposure identification number is printed on the film. At the end of the night the film is automatically exchanged from a magazine and the lens and sensors are protectively covered.

As only a single exposure is taken each night, another method is used to to record fireball event times. The total brightness of the sky is continuously monitored by a radiometer. The level and time of sudden increases in brightness are recorded with a frequency of 500 measurements/sec, providing precise light curves for all recorded events. A sound detector is simultaneously activated by the pulse of light and records for 5 minutes.

The DFO is networked by a two-way satellite internet connection. Operational logs of events and internal checks allow continuous monitoring of the camera's performance via satellite. The unit is able to inform remote operators if it detects a malfunction and software can be updated accordingly.

When a fireball is detected the camera achieves an absolute precision of 10$20 \mathrm{~m}$ for each point on the luminous trajectory and a dynamic precision of $\mathrm{ca}$. $10 \mathrm{~ms}^{-1}$. The result is high precision orbital data, as well as accurately predicted fall positions for rapid meteorite recovery. 
The main objective of the camera is a Zeiss Distagon $3.5 / 30 \mathrm{~mm}$ fish-eye lens that gives a high-resolution $180^{\circ}$ image. Images are recorded on large format $(9 \times 12 \mathrm{~cm})$ film taking a single exposure during the dark hours of clear nights (Figure 2). A three-blade revolving shutter is located above the film plate allowing the velocity of fireballs to be determined. Each loaded film is held flat by under-pressure from a vacuum pump. The camera unit contains a magazine of 32 films and, depending on the number of clear nights, human intervention is required only every 5-7 weeks to retrieve the exposed film and replace the magazine. The prototype DFO began automatic observation on 27 October 2003 and operated continuously for 2 years.

Following the test period, some modifications were made and three additional DFOs were constructed. The prototype was moved to a location in the Nullarbor Region together with two additional DFOs, and construction of the trial network now comprising four DFOs was completed in December 2007. Currently, the DFN has an effective detection coverage of ca. 200,000 sq km.

\section{THE NULLARBOR REGION AND METEORITE NOMENCLATURE}

The Nullarbor is an area of generally treeless, sparsely vegetated limestone plain situated in the south of the Australian continent where the arid zone intersects the coastline (Figure 3). The Nullarbor Region (as defined by Lowry, 1970; Beard, 1975) forms part of a larger geological formation, the Eucla Basin, containing generally flat-lying limestones of Early Miocene age, that straddles the border between Western and South Australia (Bevan and 
Binns, 1989). In Western Australia, the Eucla Basin covers an area of about $104,000 \mathrm{sq} \mathrm{km}$.

Conventionally, meteorites take the geographical names of the places where they fell, or were found. The allotted name has no scientific significance, but only serves to distinguish one meteorite from another. Naming of meteorites world-wide is governed by the Nomenclature Committee of the Meteoritical Society (http://www.meteoriticalsociety.org/bulletin/nc-guidelines.htm). Owing to the generally featureless nature of the Nullabor Region and the consequent scarcity of geographical names, a system of meteorite nomenclature (approved by the Nomenclature Committee) was devised by Bevan and Binns (1989). Using the available geographical names, 47 named areas were delineated in the Western Australian Nullarbor. Meteorite finds are assigned the name of the area in which they are found and a three digit number (e.g. 001) for each distinct meteorite (Bevan and Binns 1989). The naming system is similar to those systems currently employed to deal with the large number of meteorites being recovered from other parts of the world such as the Sahara and Antarctica. This naming/numbering system was later extended to the South Australian Nullarbor by Bevan and Pring (1993) with the delineation of an additional 27 named areas.

With the establishment of the DFN and the prospect of the recovery of a large number of observed meteorite falls (as opposed to chance finds), in accordance with guidelines set down by the Nomenclature Committee of the Meteoritical Society, unique geographical names should to be sought for 
newly recovered falls. The first recovered fall, Bunburra Rockhole, was named after a nearby geographical feature. The delineated area name of the South Australian Nullarbor in which the meteorite fell had previously been named Bunburra. As this was the first meteorite recovered from the Bunburra area, the use of the unique name presented no problem. However, subsequent recoveries of observed falls from the same, or different areas of the Nullarbor may prove difficult to name without the invention of additional names, or the use of informal local names. This nomenclature problem has yet to be addressed properly, although may be solved by using area names combined with the designated fireball number for a given fall. This will allow distinction from the more numerous chance meteorite finds on the Nullarbor.

\section{BUNBURRA ROCKHOLE, THE FIRST RECOVERED METEORITE}

The fireball associated with the first recovered meteorite fall was detected the DFN over the southern Nullarbor on 20 July, 2007 at $19 \mathrm{~h} 13 \mathrm{~m} 53.2 \mathrm{~s} \pm 0.1 \mathrm{~s}$ UT. This time marks the beginning of the fireball trajectory. The fireball designated DN200707 (Figure 4) was recorded by the two easternmost stations of the Network. The photographic record presented a far from ideal geometry because the fireball was close to the visible horizon of both stations and corresponded to angular heights in the range $9.7-5.8^{\circ}$. Nevertheless, the precision of the camera system lens and high resolution of the photographic emulsion made it possible to reduce both all-sky images and obtain precise results for atmospheric trajectory, dynamics, luminosity, orbit and predicted fall position (Spurny et al. 2008). 


\section{Atmospheric trajectory}

The fireball's luminous flight initiated at an altitude of $62.83 \mathrm{~km}$ and following a $64.7 \mathrm{~km}$ long flight path terminated at an altitude of $29.59 \mathrm{~km}$. The angle of the trajectory to the Earth's surface was $30.9^{\circ}$. The meteoroid had an initial mass of $\sim 50 \mathrm{~kg}$ and an initial velocity of $13.40 \mathrm{~km} / \mathrm{sec}$. During 5.7 secs of flight the ablated mass decelerated to a terminal velocity of $5.7 \mathrm{~km} / \mathrm{s}$. The maximum absolute (at $100 \mathrm{~km}$ distance) brightness of -9.3 magnitude was reached at an altitude of $54.8 \mathrm{~km}$. Following a slight decrease, the luminosity remained nearly constant until at the end of the trajectory three small flares were detected (Figure 5). Rapid changes in luminosity are an interesting feature of the lightcurve and are characteristic of Type I fireballs (Spurny and Ceplecha 2008).

\section{Dark flight and predicted fall position}

Computation of the dark flight of residual material was complicated by a number of factors; the end-point height of the fireball was close to $30 \mathrm{~km}$ which is relatively high; the terminal mass was predicted to be a maximum of only about $1 \mathrm{~kg}$; at the time of fall there were strong stratospheric winds up to $50 \mathrm{~m} / \mathrm{s}$ that would displace the fall position for a $100 \mathrm{~g}$ object by approximately $7.5 \mathrm{~km}$. Modelling predicted that the most probable meteoritic masses were somewhere between $100-250 \mathrm{~g}$ and these limits and the best wind model were used to obtain a fall line of highest probability. An organized ground search to recover material took place in October 2008. At the end of the first day of searching, the first meteorite with a mass of $150 \mathrm{~g}$ was found $97 \mathrm{~m}$ southward from the predicted line of fall, and a second mass of $174 \mathrm{~g}$ was 
recovered $39 \mathrm{~m}$ north of the central line. The distance between the masses was $275 \mathrm{~m}$. Later, in 2009, a third mass of $14.9 \mathrm{~g}$ was recovered.

\section{Orbital elements and analysis}

The calculated elements are; semi-major axis (AU): $0.851 \pm 0.002$; argument of perihelion: $\left({ }^{\circ}\right): 209 \pm 0.2$; eccentricity: $0.245 \pm 0.003$; longitude of ascending node $\left({ }^{\circ}\right): 297.59525 \pm 0.00010$; perihelion distance $(\mathrm{AU})$ : $0.643 \pm 0.004$; inclination $\left({ }^{\circ}\right): 9.07 \pm 0.17$; aphelion distance $(\mathrm{AU})$ : $1.05997 \pm 0.00014$; period (years): $0.786 \pm 0.003$. All angular elements are given in J2000 equinox. These results are important for two reasons. Firstly, this is one of the most precise orbits ever determined for a recovered meteorite and secondly it is an Aten-type orbit. Aten asteroids are near-Earth objects which have semi-major axes $<1$ AU. The orbit of DN200707 was almost contained within the Earth's orbit (Figure 6).

The orbit is relatively chaotic with a number of close approaches with Earth and Venus, the most recent Venusian encounter having occurred in September 2001. A search for asteroidal objects with similar orbital elements revealed several cases. However, given the chaotic nature of these orbits it is unlikely that there is a genetic relationship to the recovered meteorite. Nevertheless, as the meteorite is of an unusual type, spectral observations of these asteroids may be worthwhile.

\section{Meteorite petrology, chemistry, oxygen isotopic composition and chronology}


The meteorite, Bunburra Rockhole (Weisberg et al. 2009; Bland et al. 2009), is a brecciated basaltic achondrite (eucrite) containing numerous clasts (Figure 7). Eucrites are pigeonite-plagioclase basalts that are grouped with two other types of achondritic meteorites, the howardites (polymict breccias of basalts, gabbros and orthopyroxenites) and the diogenites (orthopyroxenites), into the HED clan of achondrites (see Mittlefehldt 2003 and references therein). Outside of the Earth, these meteorites provide the largest suite of crustal igneous rocks from a differentiated body available for study.

In Bunburra Rockhole, three texturally distinct, randomly distributed breccia lithologies are distinguished by grain size (fine- medium- and coarse-). All three grain size lithologies appear to have similar modal mineralogies. Plagioclase (52.5 vol\%) and pyroxene (41.5 vol\%) make up the essential mineralogy, with minor amounts of silica (4.5 vol\%) and traces of sulfide, ilmenite and chromite that together account for 1.5 vol\% (Benedix et al. 2010). The coarse- and medium- grained lithologies show sub-ophitic igneous textures, whereas the fine-grained lithology has a hornfels-like texture similar to the unusual eucrite, Ibitira (Steel and Smith 1976; Mittlefehldt 2005).

In Bunburra Rockhole, pyroxene is generally Fe-rich and ranges from Ca-rich to Ca-poor $\left(\mathrm{Fs}_{26.7} \mathrm{Wo}_{44.0}-\mathrm{Fs}_{63.6} \mathrm{Wo}_{2.3}\right)$. Plagioclase ranges in composition from $A n_{85}-A n_{91}$. (Bland et al., 2009). In texture, modal mineralogy, and mineral chemistry the meteorite lies within the field of 'normal' eucrites, with the exception of some pyroxene grains with $\mathrm{Fe} / \mathrm{Mn}$ ratios $>34$. However, the bulk oxygen isotope composition $\left(\Delta^{17} \mathrm{O}=-0.112\right.$ per mil.) of Bunburra 
Rockhole deviates significantly from the HED fractionation line (Bland et al. 2009). The oxygen isotopic composition plots close to another anomalous eucrite, Asuka 881394, but Bunburra Rockhole does not match that meteorite in mineral chemistry. Some aliquots of Bunburra Rockhole have oxygen isotopes similar to Ibitira. However, pyroxene compositions in Bunburra Rockhole are overall more Mg-poor than those in Ibitira. Although Bunburra Rockhole has many features of 'normal' non-cumulate eucrites, its oxygen isotopic composition indicates that it (or perhaps components of it) formed on a different parent body to that of most HED group meteorites. This may mean that there are a number of basaltic bodies with similar bulk compositions that experienced essentially similar processes but under different redox conditions, and in different regions of the Solar System.

Trace element data for pyroxene and plagioclase in the three lithologies of Bunburra Rockhole have been reported by Spivak-Birndorf et al. (2010). Essentially, The REE abundances in pyroxene are within the range of those in non-cumulate eucrites reported by Hsu and Crozaz (1996). Similarly, REE in plagioclase also lie within the range of non-cumulate eucrites. In contrast, the pyroxene phenocrysts in the fine-grained lithology of Bunburra Rockhole are are slightly depleted in REE and other trace elements relative to the coarser lithologies (Spivak-Birndorf et al. 2010).

Spivak-Birndorf et al. (2010) also investigated the Al-Mg and $\mathrm{Pb}-\mathrm{Pb}$ systematics of whole rock samples and mineral separates from Bunburra Rockhole. They report that the mineral fractions show evidence of $\mathrm{U}-\mathrm{Pb}$ 
redisribution and do not yield meaningful ages. However, two whole rock samples provided an absolute $\mathrm{Pb}-\mathrm{Pb}$ age of $4.10 \pm 0.2 \mathrm{Ga}$. Given the brecciated nature of the meteorite and the evidence of sub-solidus equilibration reported by Bland et al. (2009), Spivak-Birndorf et al. (2010) interpret the $\mathrm{Pb}-\mathrm{Pb}$ age as corresponding to a secondary heating event. Basaltic eucrites with similarly reset ages have been reported by Tera et al. (1997). The Al-Mg systematics of the meteorite reported by Spivak-Birndorf et al. (2010) indicate that $\mathrm{Mg}$ isotope equilibration occurred after the complete decay of ${ }^{26} \mathrm{Al}$ which is consistent with the young absolute $\mathrm{Pb}-\mathrm{Pb}$ age.

\section{ACCUMULATED OBSERVATIONAL DATA FROM THE DFN}

Until the operation of the DFN, reliable (e.g., photographic or video observation) meteor/fireball data were exclusively for the northern hemisphere. All observations were carried out by networks with central latitudes of $\sim 45-55^{\circ}$, so they could not observe below declinations of $-45^{\circ}$. Thus far 550 fireballs have been observed by the DFN on the Nullarbor. Double or multi-station fireball records, for which precise atmospheric trajectories and orbits can be determined, number $\sim 150$. These are the first orbits recorded for southern hemisphere fireballs. In addition, it appears very likely that a new, active meteor shower has been discovered.

In addition to Bunburra Rockhole, of the multi-station events, 11 are considered to have resulted in meteorite falls. Four of these are probable falls (10's-100 g); five are certain falls (>100's g). One of these had initial mass $\sim 20$ tonnes and may have been cometary in origin, but unfortunately it 
terminated over the Southern Ocean. One certain fall, and two probable falls, are as yet unrecovered and in searchable country. Two additional recent events are almost certain falls in searchable areas and data are still being reduced. In October 2010, a fragment $(24.54 \mathrm{~g})$ from one of these falls (DFNxxx) was recovered by a field party and marks the second success of the DFN (Bland et al., in prep).

\section{DISCUSSION}

With the recovery of Bunburra Rockhole, the DFN achieved a number of firsts. The meteorite represents only the fifth predicted fall in history. It is the first known meteorite from an Aten-type orbit, the first basaltic achondrite with a known orbit, and the first instrumentally observed fall in the southern hemisphere. It is the first meteorite recovery based on data from the newly developed instrument (DFO). Finally, it is the first documented meteorite fall from a relatively small meteoroid, that produced a weak fireball with a terminal height of $30 \mathrm{~km}$. Overall, the successful recovery is vindication of the methodological approach and instrument development. Only high resolution observations of the event could yield such a high precision orbit and accurate impact site data for what was a relatively unfavourable case.

Considering the possible asteroidal source of Bunburra Rockhole, a number of workers (e.g. Binzel and Xu, 1993; Burbine et al., 2001) have investigated the spectral similarities between asteroid 4Vesta, Vestoids (small asteroids with spectral characteristics similar to that of $4 \mathrm{Vesta}$ ) and HED meteorites, noting the dynamical orbital association of 4 Vesta and the Vestoids, and 
potential delivery paths to Earth. To date there are no orbital data for HED related meteorites. On the basis of the recovery of Bunburra Rockhole, a basaltic eucrite delivered to Earth from an Aten-type orbit, the question arises, what if any are the possible relationships with the V-type asteroids/HED meteorite association?

From the Near Earth Object (NEO) model (Bottke et al. 2002) predictions of the probability can be made that particular NEOs came from different source regions using their present day $a, e$ and $i$ values. On the assumption that small NEOs have the same orbital distribution as the observed km-sized NEOs, then the probability of the Bunburra Rockhole meteorite came from the innermost region of the Main Belt of asteroids is $98 \%$ (Bland et al. 2009). A $72 \%$ probability exists that it was delivered from the $v_{6}$ resonance (the inner edge of the Main Belt of asteorids near 2AU at low inclinations), and a $26 \%$ probability that it was delivered from the large number of resonances located in the inner Main Belt. A further possibility that it came from the 3:1 Kirkwood gap (between $2.45-2.55 \mathrm{AU}$ ) has a probability of only $3 \%$. What is certain is that the meteorite did not come from the outer Main Belt >2.8AU. Arguments have been presented favouring the 3:1 resonance (Binzel and Xu 1993; Binzel et al. 1999), the $v_{6}$ (Migliorini et al. 1997) or both (Vilas et al. 2000) as resonances that are plausible for Vesta-derived ejecta. For Bunburra Rockhole the $v_{6}$ resonance is favoured (Bland et al. 2009).

If 4 Vesta really is the source of the HED clan of meteorites (e.g. see Sykes and Vilas 2001; Pieters et al. 2005) then the anomalous oxygen isotopic 
composition of Bunburra Rockhole indicates that it is highly unlikely that it is derived from 4 Vesta. The meteorite joins a small group of anomalous basaltic achondrites characterized by unusual oxygen isotope compositions. The first to be identified was North West Africa (NWA) 011 (Yamaguchi et al. 2002) followed by a further five anomalous basaltic achondrites (Scott et al. 2008 and 2009; Weichert et al. 2004; Mittlefehldt, 2005; Lenz et al. 2007). Some of these meteorites have unusual mineral compositions such as Ibitira (Mittlefehldt, 2005; Lenz et al. 2007) while others, like Asuka 881394, have anomalous ages (Wadhwa et al. 2005). Of the six meteorites known to date, only four (Ibitira, (NWA) 011, Asuka 881394 and Bunburra Rockhole) diverge sufficiently from the HED mass fractionation line to require parent asteroids that are different to 4Vesta.

With the exception of oxygen isotopes, in most respects Bunburra Rockhole mineralogically resembles other brecciated basaltic eucrites. Future work on Bunburra Rockhole will include the determination of mineral-specific oxygen isotopic compositions to locate the source of the anomalous oxygen isotopes. Trace element mapping and Fe-oxidation state determination using the Australian synchrotron may also reveal any anomalous component of the meteorite.

Having proved the potential of the DFN concept (Figure 8), the near-term goal now is an expansion to a much wider network covering 1 million sq $\mathrm{km}$ of southern Australia. A large dataset of meteorites with orbits could provide answers to many important unanswered questions. For example, do any 
meteorites originate from comets; do rare meteorites have unusual orbits; do streams of meteorites exist; generally do meteorites come directly from the main belt of asteroids, or from near-Earth parent asteroids like Bunburra Rockhole? Moreover, is it possible, with precise orbital information, to link a meteorite with a short cosmic ray exposure age to a particular parent asteroid thus effectively providing a free sample-return mission for that body? Finally, is the compositional structure of the asteroid belt as revealed by IR spectroscopy reflected in meteorite composition?

\section{ACKNOWLEDGEMENTS}




\section{REFERENCES}

BEARD J. S. 1975. Nullarbor, 1:1,000,000 Vegetation Series, Explanatory notes to sheet 4. The Vegetation of the Nullarbor area. In: Vegetation Survey of Western Australia, University of Western Australia Press, 104pp.

BENEDIX G. K., BLAND P. A., HOWARD K. T., SPURNY P. and BEVAN A. W. R. 2010 Mineralogy and Petrology of Bunburra Rockhole. 41st Lunar and Planetary Science Conference, 1438.pdf

BEVAN A. W. R. 2006. Desert meteorites; a history (In: McCall G.J.H., Bowden A.J. \& Howarth R.J. eds.), The history of meteoritics and key meteorite collections; fireballs, falls and finds. Geological Society, London, Special Publications 256, 325-343.

BEVAN A. W. R. \& BINNS R. A. 1989. Meteorites from the Nullarbor Region, Western Australia: I. A review of past recoveries and a procedure for naming new finds. Meteoritics, 24, 127-133.

BEVAN A. W. R. \& PRING A. 1993. Guidelines for the naming of new meteorite finds from the Nullarbor Region, South Australia. Meteoritics 28, 600-602.

BINZEL R.P., BUS S.J., \& BURBINE T.H. 1999, $30^{\text {th }}$ Lunar and Planetary Science Conference abstract \#1216.

BINZEL R. P. \& XU S. 1993. Chips off of Asteroid 4 Vesta: Evidence for the parent body of basaltic achondrite meteorites. Science 260, 186-191. BLAND P. A., SPURNY P., TOWNER M. C., BEVAN A. W. R., SINGLETON A., BOTTKE W. F., Jr., GREENWOOD R. C., CHESLEY S. R., SHRBENY L., BOROVICKA J., CEPLECHA Z., McCLAFFERTY T. P., VAUGHAN D., BENEDIX G. K., DEACON G., HOWARD K. T., FRANCHI I. A., \& HOUGH R. 
M. 2009. An Anomalous Basaltic Meteorite from the Innermost Main Belt. Science 325, 1525-1527.

BOWDEN A. J. 2006. Meteorite provenance and the asteroid connection. (In: McCall, G.J.H., Bowden, A.J. \& Howarth, R.J. eds.), The history of meteoritics and key meteorite collections; fireballs, falls and finds. Geological Society, London, Special Publications 256, 379-403.

BURBINE. 2001.

HALLIDAY I., BLACKWELL A. T. \& GRIFFIN A. A. 1989. The flux of meteorites on the Earth's surface. Meteoritics 24, 173-178.

HSU W. \& CROZAZ G. 1996. Mineral chemistry and the petrogenesis of eucrites. I. Noncumulate eucrites. Geochimica et Cosmochimica Acta 60, 4571-4591.

JENNISKENS P., SHADDAD M. H., NUMAN D., ELSIR S., KUDODA A. M., ZOLENSKY M. E., LE L., ROBINSON G. A., FRIEDRICH J. M., RUMBLE D., STEELE A., CHESLEY S. R., FITZSIMMONS A., DUDDY S., HSIEH H. H., RAMSAY G., BROWN P. G., EDWARDS W. N., TAGLIAFERRI E., BOSLOUGH M. B., SPALDING R. E., DANTOWITZ R., KOZUBAL M., PRAVEC P., BOROVICKA J., CHARVAT Z., VAUBAILLON J., KUIPER J., ALBERS J., BISHOP J. L., MANCINELLI R. L., SANDFORD S. A., MILAM S. N., NUEVO M. \& WORDEN S. P. 2009. The impact and recovery of asteroid $2008 \mathrm{TC}_{3}$. Nature 458, 485-488.

LENZ R. C. F., SCOTT E. R. D. \& McCoy T. J. 2007. Amomalous eucrites: Using Fe/Mn to search for different parent bodies. $38^{\text {th }}$ Lunar and Planetary Science Conference 1968.pdf.

LOWRY D. C. 1970. Geology of the western part of the Eucla Basin. 
Geological Survey of Western Australia Bulletin 122, 1-181.

MIGLIORINI F., MORBIDELLI A., ZAPPAL V., GLADMAN B. J., BAILEY M. E.

\& CELLINO A. 1997. Vesta fragments from 6 and 3:1 resonances:

Implications for V-type near-Earth asteroids and howardite, eucrite, and

diogenite meteorites. Meteoritics \& Planetary Science 32, 903-916.

MITTLEFEHLDT D. W. 2003. Achondrites. In Meteorites, Comets and Planets (ed. Davis A. M.) Vol.1 Treatise on Geochemistry (eds. Holland H. D. \&

Turekian K. K.), Elsevier-Pergamon, Oxford, 291-324.

MITTLEFEHLDT D. W. 2005. Ibitira: A basaltic achondrite from a distinct parent asteroid and implications for the Dawn mission. Meteoritics \& Planetary Science 40, 665-677.

PIETERS C. M., BINZEL R. P., BOGARD D., HIROI T., MITTLE FEHLDT D. W., NYQUIST L., RIVKIN A. \& TAKEDA H. 2005. Asteroid-meteorite links: the Vesta conundrum(s). Asteroids, Comets, Meteors Proceedings of the IAU Symposium (Lazzara D., Ferraz-Mello S. \& Fernández J. A. eds.) 229, 273288.

SCOTT E. R. D., GREENWOOD R. C., FRANCHI, I. A., BARRAT J-A. \& SANDERS I. 2008. Oxygen isotopic constraints on the number and origin of basaltic achondrite parent bodies. $39^{\text {th }}$ Lunar and Planetary Science Conference 2344.pdf.

SCOTT E. R. D., GREENWOOD R. C., FRANCHI I. A. \& SANDERS, I. A. (2009). Oxygen isotopic constraints on the origin and parent bodies of eucrites, diogenites, and howardites. Geochimica et Cosmochimica Acta, 73, $5835-5853$. 
SPIVAK-BIRNDORF L. J., BOUVIER A., WADWHA M., BLAND P. A. and SPURNY P. 2010. Trace element geochemistry and chronology of the Bunburra Rockhole basaltic achondrite. 41st Lunar and Planetary Science Conference, 2274.pdf

SPURNY P., BLAND P. A., BOROVICKA J., SHRBENY L., McCLAFFERTY T., SINGLETON A., BEVAN A. W. R., VAUGHAN D., TOWNER M. C. \& DEACON G. 2009. The Bunburra Rockhole meteorite fall in SW Australia: Determination of the fireball trajectory, luminosity and impact position from photographic records. $40^{\text {th }}$ Lunar and Planetary Science Conference, 1498.pdf

SPURNY P., BOROVICKA J. \& SHRBENY L. 2007. Automation of the Czech part of the European fireball network: equipment, methods and first results In: Near Earth Objects, our Celestial Neighbors: Opportunity and Risk (eds. Milani A., Valsecchi G.B. \& Vokrouhlicky D.), Cambridge Univ. Press. Proceedings IAU Symposium No. 236,121-130.

SPURNY P. \& CEPLECHA Z. 2008.

STEELE I. M. \& SMITH J. V. 1976. Mineralogy of the Ibitira eucrite and comparison with other eucrites and lunar samples. Earth and Planetary Science Letters 33, 67-78.

SYKES M. V. \& VILAS F. 2001. Closing in on HED meteorite sources. Earth Planets Space 53, 1077-1083.

TERA F., CARLSON R. \& BOCTOR N. 1997. Radiometric ages of basaltic achondrites and their relation to the early solar system. Geochimica et Cosmochimica Acta 61, 1713-1731.

VILAS F., COCHRAN A. L., \& JARVIS K. S. 2000. Vesta and the Vestoids: A 
new rock group?, Icarus, 147, 119-128.

WADHWA et al. 2005.

WIECHERT U. H., HALLIDAY A. N. PALME H. \& RUMBLE D. 2004. Oxygen isotope evidence for rapid mixing of the HED meteorite parent body. Earth \& Planetary Science Letters 221, 373-382.

WEISBERG et al. 2009.

YAMAGUCHI A., CLAYTON R. N., MAYEDA T. K., EBIHARA M., OURA Y., MIURA Y. N., HARAMURA H., MISAWA K., KOJIMA H. \& NAGAO K. 2002. A new source of basaltic meteorites inferred from North West Africa 011. Science 296, 334-336.

ZOLENSKY M. E. 1998. The flux of meteorites to Antarctica. (In: Grady M. M., Hutchison R., McCall G. J. H. \& Rothery D. A. eds.) Meteorites: Flux with Time and Impact Effects. Geological Society, London, Special Publications 140, 93-104. 
Figure captions

Figure 1. Prototype DFO unit during construction.

Figure 2. All-Sky image showing a fireball crossing star trails around the South Celestial Pole. The star trails indicate an exposure time of around $3 \mathrm{hrs}$. The image of the fireball is segmented by the camera shutter operating at a know rate allowing the velocity of the object to be determined.

Figure 3. Location of the Nullarbor Region and typical terrain (inset).

Figure 4. All-Sky image of the fireball (DN200707) associated with the fall of the Bunburra Rockhole meteorite.

Figure 5. Lightcurve of the fireball (DN200707) from DFO 02 of the Desert Fireball Network.

Figure 6. Orbit of the meteoroid that gave rise to DN/Bunburra Rockhole meteorite fall projected on the plane of the ecliptic.

Figure 7. Mass of the Bunburra Rockhole meteorite at the site of discovery and (inset) a BSE image showing $\mathrm{F}=$ fine grained, $\mathrm{M}=$ =medium grained and $\mathrm{C}=$ coarse grained, lithologies.

Figure 8. Fully operational solar powered DFO station of the Desert Fireball Network with satellite link. 